\title{
EP3 Receptor Deficiency Attenuates Vascular Remodeling and Cognitive Impairment in a Cerebral Small Vessel Disease Model
}

$\mathrm{Na}$ Liu

Fudan University Huashan Hospital Department of Neurology

Jie Tang

Huashan Hospital Fudan University

Yang Xue

Huashan Hospital Fudan University

Miaoyi Zhang

North Huashan Hospital Fudan University

Xue Ren

Huashan Hospital Fudan University

Vincent Mok

the Chinese University of Hong Kong

Yilong Wang

Beijing Tiantan Hospital Department of Neurology

Jianhui Fu ( $\nabla$ jianhuifu@126.com )

Huashan Hospital Fudan University

\section{Research}

Keywords: cerebral small vessel disease, hypertension, E prostanoid receptor 3, transforming growth factor beta1, extracellular matrix

Posted Date: October 26th, 2020

DOl: https://doi.org/10.21203/rs.3.rs-95403/v1

License: (c) (i) This work is licensed under a Creative Commons Attribution 4.0 International License. Read Full License 


\section{Abstract}

\section{Background}

Anti-hypertensive therapy has achieved effective but not complete results in treating cerebral small vessel disease (CSVD), suggesting the necessity for additional treatments. Targeting abnormal inflammatory responses has become a research interest. Inhibition of E-prostanoid 3(EP3) receptor has been shown to attenuate vascular remodeling in peripheral organs, but little is known about its role in CSVD. Here, we investigated whether the deletion of EP3 attenuates the development of CSVD.

\section{Methods}

Stroke-prone renovascular hypertensive rat (RHRsp) was used as an animal model of CSVD. The effect of EP3 deletion on the expression of extracellular matrix (ECM) in cerebral small arteries of RHRsp was detected by immunofluorescence. Changes in cognitive function and cerebral blood flow (CBF) of RHRsp were evaluated using the Morris water maze test and MRI. In vitro experiments with primary rat brain microvascular smooth muscle cells were used to confirm whether the transforming growth factor beta 1 (TGF- $\beta 1$ ) signal takes part in the process

Results

Cerebral small arteries of RHRsp exhibited increased EP3 expression. Despite no alleviation in hypertension, the deletion of EP3 still reduced the overexpression of ECM in the cerebral small arteries of RHRsp. In vitro experiments indicated that EP3 deletion regulated the expression of ECM by downregulating TGF- $\beta 1 /$ Smad signaling. EP3 knockout further attenuated the cognitive impairment of the RHRsp possibly through increased CBF.

\section{Conclusion}

Together, our results indicate that the deletion of EP3 attenuates the vascular remodeling and vascular cognitive impairment induced by hypertension, and blockade of the EP3 receptor may be a promising strategy for the treatment of CSVD.

\section{Background}

Cerebral small artery remodeling is a main pathological feature of arteriolosclerotic cerebral small vessel disease (CSVD), characterized by lumen narrowing and abnormal accumulation of extracellular matrix $(E C M)$ in the vessel wall[1]. Cerebral small artery remodeling reduces cerebral blood flow (CBF) leading to hypoperfusion of the brain [2,3], which is closely related to the subsequent development of cognitive impairment [4, 5]. Hypertension is a major risk factor for CSVD[6], and chronic hypertension triggers vascular remodeling in CSVD. However, recent clinical studies also reveal that despite anti-hypertensive medication, some patients still present progressive vessel pathology and cognitive disorder[7, 8]. Given 
this, additional treatment apart from anti-hypertension targeting vascular remodeling in CSVD is becoming increasingly important.

Inflammation has long been listed as a candidate factor in the development of CSVD[9], and tackling abnormal inflammatory responses has become a potential treatment target for CSVD. One of the research focuses is cyclooxygenase 2 (COX-2), a key enzyme in prostaglandin biosynthesis. A study showed that a COX-2 genetic polymorphism in the Chinese population may contribute to the risk of developing white matter lesions[10]. Our previous study also revealed that using COX-2 inhibitor can attenuate CSVD in an animal model[11]. However, chronic use of COX-2 inhibitors also results in adverse side effects and increases the risk of vascular diseases possibly by inhibiting the endothelial prostacyclin production[12], leading us to consider that modulating the prostaglandins and prostaglandin receptors downstream of the COX-2 pathway would be a much better strategy.

Among the prostaglandins synthesized from COX-2, prostaglandin E2 $\left(\mathrm{PGE}_{2}\right)$ is the main product produced during inflammatory processes. $\mathrm{PGE}_{2}$ has four receptor subtypes, namely, E prostanoid (EP) receptors (EP1-4) [13]. Elevated expression of EP3 receptors has been reported in the small arteries of hypertensive animals[14], and inhibition of EP3 receptors has been shown to reverse cardiac hypertrophy induced by hypertension[15] or attenuated pulmonary arteriole remodeling in pulmonary hypertension[16]. However, the role of EP3 receptors in the cerebral small artery remodeling of CSVD induced by hypertension is still unknown.

With this background, this study aimed to evaluate the role of EP3 receptors in CSVD, using a renal hypertensive animal model- stroke-prone renovascular hypertensive rat (RHRsp). The RHRsp model is established by a two-kidney, two-clip(2k2c) procedure[17]. After the operation, RHRsp presents with persistent hypertension. Studies have shown that RHRsp rats presented cerebral small artery remodeling with fibrinoid necrosis, hyalinosis, and apparent luminal narrowing[18]. Behavioral tests also confirmed cognitive impairment in RHRsp[19]. These results suggest that RHRsp is a relatively ideal animal model of CSVD, especially for the study of vascular pathological changes in hypertension-associated CSVD.

\section{Methods}

\section{Animals and Treatment}

Because of the possible effect of sex hormones on hypertension induced by two-kidney, two-clip (2k2c) procedure, experiments were performed in male rats. Animals were housed in rooms under conditions of controlled temperature $\left(21 \pm 2^{\circ} \mathrm{C}\right)$, humidity $(60 \pm 10 \%)$, and a $12 \mathrm{~h}$ light/dark cycle. $E P 3^{-/-}(E P 3$ knockout $)$ rats in a Sprague-Dawley (SD) background were created by Beijing Biocytogen (Beijing, China) using CRISPR/Cas9-based technology (detailed description provided in the Expanded Materials and Methods). Wild-type (WT) littermates (EP3 ${ }^{+/+}$rats) were generated as experimental controls from EP3 receptor heterozygous mating. We used $30 \mathrm{EP}^{-/-}$rats and $30 \mathrm{WT}$ rats in this study. Rats were subjected to either a two-kidney, two-clip (2k2c) procedure to establish the RHRsp model or a sham surgery for control group. 
The operation details are provided in the Supplementary Material. Investigators responsible for functional assessment, outcome measurement, and data analysis were blinded to experimental groups.

\section{Blood Pressure (BP) monitoring}

$\mathrm{BP}$ measurements were performed in adult $\mathrm{EP}^{-/-}$and WT rats aged 12 weeks. Two months and four months after the $2 \mathrm{k} 2 \mathrm{c}$ procedure or sham operation, the BP of the animals was also measured.

Conscious BP measurement using the tail-cuff method (BP-98A; Softron Co, Tokyo,Japan): the day before blood pressure was measured, animals were induced into the holding cage for $15 \mathrm{~min}$ in order to help the animals adapt to the environment. On the day of measurement, the instrument was preheated for $15 \mathrm{~min}$ before use, and then the rats were induced into the holding cage, with its tail being properly put into the pressurized sensor sleeve. Blood pressure was measured only after the animals calm down in the cage. The measurement was repeated three times at intervals greater than five min, and the average of each animal was taken. Unconscious BP measurement using invasive carotid artery catheterization: the rats were anesthetized with sodium pentobarbital and placed on a temperature-controlled pad. One side of the carotid artery was exposed and a polyethylene tube prefilled with heparinized normal saline was inserted into the artery, and BP was measured by a pressure transducer and recorded in real-time.

\section{Evaluation of CBF and Cognitive Function}

Dynamic susceptibility contrast perfusion-weighted imaging (DSC-PWI) was conducted to evaluate the $\mathrm{CBF}$ of animals. The Morris water maze test was conducted 16 weeks after the $2 \mathrm{k} 2 \mathrm{c}$ operation to evaluate the cognitive function of the animals. The detailed methods are provided in the Supplementary Material.

\section{Brain Tissue Preparation}

The rats were sacrificed 4-5 months after surgery and transcardially perfused with approximately $300 \mathrm{~mL}$ of $0.9 \%$ saline, followed by $200 \mathrm{~mL}$ of $4 \%$ paraformaldehyde (PFA) in $0.1 \mathrm{M}$ phosphate buffered saline (PBS, pH 7.4) within $5 \mathrm{~min}$. Then, the brain tissues were post-fixed in 4\%PFA for 4-6h and stored in 30\% sucrose in $0.1 \mathrm{M}$ PBS (pH 7.4) until the brain tissues fell to the bottom of the solution. The brains were sectioned into coronal serial slices (10 $\mu \mathrm{m}$ thickness) from about $+5 \mathrm{~mm}$ bregma to $-5 \mathrm{~mm}$ bregma.

\section{Immunofluorescence}

Primary antibodies against EP3(Sigma), smooth muscle actin alpha (aSMA, Sigma), collagen I (Abcam), collagen IV (Abcam), fibronectin (Abcam) and laminin (Abcam) were used. The slides were photographed using a fluorescence microscope (Olympus, BX-51, Tokyo, Japan). For each primary antibody, three sections per animal were stained. We divided each cross-section of the brain into three parts according to a previous study[20]: cortical gray matter, white matter, and deep gray matter. At least three random fields of each part were selected and photographed for further analysis. The cerebral small arteries $(10-65 \mu \mathrm{m})$ 
were indicated by the immunostaining of aSMA[19]. The detailed methods are provided in the Supplementary Material.

\section{Cell culture}

Primary rat brain microvascular smooth muscle cells (BMVSMCs) were cultured from male 5-day-old rats by modification of the procedure described previously[21, 22] (Detailed description provided in the Supplementary Material). Immunocytochemistry with anti-aSMA was used for the identification of vascular smooth muscle cells(VSMCs). Before stimulation, rat BMVSMCs were growth-arrested by incubation in serum-free medium for $24 \mathrm{~h}$. Angiotensin II (ANGII, Sigma), LY364947 (Selleck), and L798106 (Cayman) were used for the stimulation.

\section{Western blotting}

The concentration of proteins extracted from rat BMVSMCs was determined using a Pierce BCA Protein Assay Kit (Pierce, Rockford, IL). Equal quantities of proteins were denatured and resolved by $10 \%$ SDSPAGE, transferred to nitrocellulose membranes, incubated with $5 \%$ skimmed milk for $1 \mathrm{~h}$, and then incubated with primary antibodies overnight at $4^{\circ} \mathrm{C}$. The membranes were then conjugated with a horseradish peroxidase-labeled secondary antibody for $1 \mathrm{~h}$ at RT. Blots were developed using an enhanced chemiluminescence reagent (Thermo Scientific), followed by densitometric quantification using ImageJ.

\section{Statistical Analysis}

Prism 8.0 software was used for data analysis. Data are presented as the mean \pm standard deviation. All data sets were normally distributed, and variance was homogeneous among all groups as determined by one-way analysis of variance. Differences between two groups were analyzed using a t-test and data among multiple groups were compared using one-way analysis of variance. Statistical significance was set at $p<0.05$.

\section{Results}

\section{EP3 is upregulated in cerebral small arteries of RHRsp, and deletion of EP3 does not affect blood pressure}

COX-2/ PGE2 signaling is an important inflammatory pathway. Hypertension is strongly associated with the augmented expression of the COX-2/ PGE 2 /EP3 axis in both human and animal models[14, 15]. As such, we also observed significantly elevated expression of EP3 in the cerebral small arteries of RHRsp by immunofluorescence (Figure 1A), indicating that the PGE2/EP3 axis might be involved in the progression of CSVD induced by hypertension.

We then constructed the RHRsp model on EP3 knockout rats $\left(E P 3^{--}\right)$to determine whether EP3 receptor deficiency can attenuate the development of CSVD. First, we sought to determine whether the deletion of 
$E P 3$ can affect the BP of the animals since hypertension is a crucial risk factor for CSVD and the PGE 2 signaling is reported to have major effects on BP control through its receptors[23]. We used two methods to monitor BP, including conscious BP measurement using the tail-cuff method and unconscious BP measurement using the invasive carotid artery catheterization. Results showed no significant difference in the baseline BP of either group (Figure 1B). Furthermore, we measured the BP of the rats after the 2k2c procedure. Both $E P 3^{\%}$ rats and the WT rats showed significantly increased BP compared to the shamoperated groups. However, there was still no difference between $E P 3^{--}$rats and the WT rats within the hypertensive group (Figure 1B), indicating that EP3 knockout did not impact both baseline BP and the induction of hypertension by the $2 \mathrm{k} 2 \mathrm{c}$ procedure.

\section{Deletion of EP3 attenuates the overexpression of ECM in the cerebral small arteries of RHRsp}

Next, we tested whether disruption of EP3 modulates the progression of cerebral small artery remodeling in RHRsp. Two months after the 2k2c procedure, rats in the RHRsp group developed stable hypertension. By the end of 4-5months, the cerebral small arteries of RHRsp showed a significantly higher expression of ECM including fibronectin, laminin, collagen I, and collagen IV, compared with that observed in controls with normal BP. Intriguingly, $E P 3^{-/-}$RHRsp rats displayed a significant reduction in all four kinds of ECM, compared with that in WT RHRsp (Figure 2). These results indicated that despite no effect on hypertension, the deletion of EP3 still attenuated the overexpression of ECM in the cerebral small arteries of RHRsp.

\section{Deletion of EP3 decreases the expression of ECM in BMVSMCs under Angll stimulation}

As shown in above, our results confirmed that the deletion of EP3 successfully attenuated cerebral artery remodeling in RHRsp without alleviating high blood pressure. Hence, we assumed that EP3 may regulate the expression of ECM directly by affecting VSMCs. To further study the mechanism by which EP3

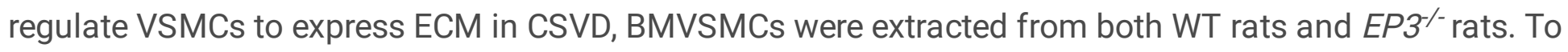
mimic the elevated expression of ECM in vitro, we used ANGIl to stimulate BMVSMCs, since both hypertensive humans and animals including RHRsp were reported to have an elevated level of ANGII[24, 25]. As shown in Figure 3, under the stimulation of ANGII, BMVSMCs exhibited significantly higher ECM expression including fibronectin, laminin, collagen I, and collagen IV. We found that the addition of the EP3 inhibitor L798106 could significantly alleviate this increased ECM expression induced by ANGII. Furthermore, the deletion of EP3 genes can significantly inhibit the baseline expression of ECM (without ANGII stimulation), and even under the stimulation of ANGII, a significantly higher expression of ECM was not observed in $E P 3^{-/}$rat BMVSMCs. These results suggest that $E P 3$ gene knockout can reverse the increased expression of ECM induced by ANGII in rat BMVSMCs.

Deletion of EP3 decreases the expression of ECM by suppressing TGF- $\beta 1$ signaling in BMVSMCs under ANGII stimulation 
TGF- $\beta 1$ signaling has long been reported to play a role in regulating ECM expression[26]. It has also been suggested that knockout of EP3 downregulates the expression of ECM in pulmonary arterioles by suppressing the TGF- $\beta 1 /$ Smad pathway[16]. In this study, we sought to determine whether EP3 can regulate the expression of ECM by altering the TGF- $\beta 1$ signaling. As shown in Figure 4A-B, western blot analyses confirmed that the level of TGF- $\beta 1$ and phosphorylation of Smad2/3 were elevated in rat BMVSMCs under ANGII stimulation. Inhibiting TGF- $\beta 1$ signaling using LY364947 attenuated the high expression of ECM induced by ANGII in rat BMVSMCs (Figure 4A). Furthermore, the deletion of EP3 rescued ANGII-induced activation of TGF- $\beta 1$ signaling, as evidenced by a decreased level of TGF- $\beta 1$ and phosphorylation of Smad2/3(Figure 4B). These results indicated that the deletion of EP3 can decrease the expression of ECM by suppressing TGF- $\beta 1$ signaling in BMVSMCs under ANGIl stimulation.

Previous studies have shown that the EP3 receptor modulates multiple intracellular signaling pathways by coupling different types of heterotrimeric $G$ proteins[27]. As shown in Figure $4 C$, the downregulation of ECM by EP3 inhibition was abolished by pretreatment with pertussis toxin (PTX) in BMVSMCs, indicating that EP3 signaling regulated the expression of ECM via the PTX-sensitive G protein, Gi/o in BMVSMCs.

\section{Deletion of EP3 attenuates decreased CBF in RHRsp}

Cerebral small artery remodeling is the leading cause of decreased CBF in patients[28]. In order to determine whether attenuated vascular remodeling caused by EP3 deletion can further improve CBF in RHRsp, we used PWI to monitor the global CBF of the animals. T2-weighted images and representative $\mathrm{CBF}$ images of the animals are shown in Figure 5. Consistent with previous studies on $E P 3^{-/}$mice[29] or mice treated with selective antagonists of EP3[30], our results showed no difference in CBF between normal WT rats and EP3\% rats. As decreased CBF is seen in CSVD patients[31], our results showed that RHRsp demonstrated a lower global CBF, compared to the control rats. Intriguingly, $E P 3^{-/-}$RHRsp rats displayed a recovery of global CBF, compared with that in WT RHRsp. These results indicate that the deletion of EP3 can improve CBF in RHRsp, confirming our hypothesis that the deletion of EP3 can not only reverse vascular structure changes but also contribute to the functional recovery of blood supply in the brain.

\section{Deletion of EP3 attenuates cognitive impairment in RHRsp}

As seen above, the deletion of EP3 successfully attenuated vascular remodeling in RHRsp and improved the cerebral blood supply of the animals. We assume that these improvements may also help to attenuate the cognitive impairment in RHRsp. To this end, we used the Morris water maze test to evaluate the cognitive function of the animals. The Morris water maze test is designed to reflect the spatial learning and memory ability. During the hidden platform acquisition phase, on average, rats in all groups showed a progressive decrease in escape latency, indicating that subjects could learn the location of the platform. However, compared with the sham-operated group, RHRsp rats had a longer escape latency, and a statistical difference appeared on the third and fifth days. The deletion of EP3 (EP3-1/RHRsp) shortened the escape latency. However, there was no statistically significant difference (Figure 6). A no-platform 
probe test was conducted $24 \mathrm{~h}$ after the final day 5 training trial. Figure 6 shows that the time spent in the target quadrant was decreased in RHRsp rats, compared with the sham-operated group. The deletion of $E P 3\left(E P 3^{-/-} \mathrm{RHRsp}\right)$ reversed this effect. The average number of times crossing the platform was higher in RHRsp than in sham-operated rats, and the deletion of $E P 3\left(E P 3^{-/-} \mathrm{RHRsp}\right)$ significantly improved the number. Taken together, these results show damaged spatial learning and memory ability in RHRsp, and the deletion of EP3 in RHRsp can rescue cognitive impairment. We cautiously speculate that the improvement of cognitive function in $E P 3^{-/}{ }^{-}$RHRsp could be partially attributed to the reduced vascular remodeling and increased $C B F$ by the deletion of EP3.

\section{Discussion}

In this study, we first observed increased EP3 expression in the cerebral small arteries of an animal model of CSVD. Then, we demonstrated that EP3 gene knockout can reverse cerebral small artery remodeling, possibly by downregulating TGF- $\beta 1$ signaling. Furthermore, we found that vascular structure improvement by EP3 deletion also attenuates the decreased CBF of RHRsp and eventually yield success in reducing the cognitive impairment of the animals.

Our results revealed the possibility to improve vascular remodeling in CSVD through EP3 deletion, while independent of controlling hypertension. Increased $C O X-2 / \mathrm{PGE}_{2} / \mathrm{EP}_{3}$ signaling has long been observed in hypertensive patients and animals, and inhibition of the signaling pathway attenuates the vascular remodeling in hypertension $[14,15]$. Although $C O X-2 / P G E_{2} / E P 3$ signaling is found to affect $B P[23]$, the conclusion is conflicting on whether the improvement of vascular remodeling by inhabitations of COX$2 / \mathrm{PGE}_{2} / \mathrm{EP} 3$ signaling in vivo is accompanied by a decrease in hypertension[11, 15, 32]. The possible reasons are that different hypertension induction methods are used in these studies and the genetic backgrounds of the animals are distinct[32]. In this study, our results indicated that EP3 knockout did not affect the baseline BP of the rats, and previous studies on EP3\%- mice suggest the same outcome[29, 33]. Although the EP3 gene knockout was reported to relieve hypertension caused by ANGII perfusion in mice[34] or salt-induced hypertension in mice[33], our results suggest that EP3 gene knockout did not affect the hypertensive response induced by the $2 k 2 c$ operation. These results may indicate that the mechanism of persistent hypertension in RHRsp is much more complex. After the surgery, as juvenile rats grow, the renal arteries were gradually narrowed by the fixed-size silver clamps, gradually making the rats' bilateral kidneys ischemic. Continuous renal ischemia activates the renin-angiotensin system and increases the release of sympathetic neurotransmitters, endothelin, aldosterone, and other substances, thereby maintaining the persistence of hypertension[17, 25]. Our results further confirm the existence of hypertension independent mechanisms behind vascular remodeling in hypertension-induced CSVD, which are promising targets for CSVD treatments.

Since the deletion of EP3 attenuated the ECM over-expression in the cerebral small arteries of $E P 3^{-1-}$ RHRsp without relieving hypertension, we assumed that direct regulation of VSMCs by the EP3 gene could be crucial in the process. Our in vitro study later confirmed that EP3 can directly regulate the 
expression of ECM in VSMCs under the stimulation of Angll. A similar effect of EP3 on VSMCs has already been reported in pulmonary arteriolar smooth muscle cells[16], and research has also suggested that EP3 gene knockout can reduce the migration and polarity of VSMCs[35]. TGF- $\beta 1$ signaling has long been found to regulate ECM production. Studies have indicated an elevated level of TGF- $\beta 1$ in both hypertensive patient[36] and hypertensive animal models[37]. Our results demonstrated that the level of TGF- $\beta 1$ and phosphorylation of Smad2/3 were elevated in rat BMVSMCs under ANGII stimulation and were relevant to the high expression of ECM. The deletion of EP3 can reverse increased TGF- $\beta 1 / \mathrm{Smad}$ signaling and attenuate the high expression of ECM. Previous studies also revealed that different receptors of $\mathrm{PGE}_{2}$ (EP1-4) participate in the regulation of ECM expression by affecting TGF- $\beta 1 /$ Smad signaling. For example, studies have shown that the TGF- $\beta 1$ pathway is involved in regulating the expression of renal vascular ECM, which is closely related to the occurrence of renal fibrosis. Activation of EP1 and EP3 receptors promotes renal fibrosis and accelerates renal damage[38], while activation of EP2[39] and EP4[40] receptors can inhibit TGF- $\beta 1$-mediated increased expression of ECM in renal mesangial cells and alleviate renal damage. $\mathrm{PGE}_{2}$ can also inhibit TGF- $\beta 1$ /Smad signaling via the EP2 receptor, thereby reducing the expression of various types of collagen as well as the formation of hypertrophic scars[41]. These results suggest that the role of the COX2/PGE $2 / E P$ axis in vascular remodeling in diseases is a complex issue. In different tissues and organs or under different pathological conditions, the COX2/PGE 2 axis may regulate ECM expression through TGF- $\beta 1$ signaling by binding to different receptors.

In our study, the deletion of EP3 further improved the CBF and cognitive function in RHRsp. We assume that this could be partially attributed to reduced vascular remodeling under EP3 deletion. However, our research cannot rule out other possible mechanisms in the process. $\mathrm{PGE}_{2}$ acts as a vasodilator and vasodepressor through four distinct EP receptors, and stimulation of EP3 triggers processes leading to vasoconstriction[42]. It has been reported that activation of EP3 triggers vasoconstriction in porcine middle cerebral arteries[43] and in the guinea pig aorta[44]. It is possible that EP3 deletion contributed to the improvement of CBF in RHRsp by reversing the enhanced vascular constriction under hypertension. Our study also witnessed an improved cognitive function accompanied by an increased $\mathrm{CBF}$ in $\mathrm{EP} 3^{-1-}$ RHRsp. In addition to vascular protection, EP3 deletion may preserve cognitive function in RHRsp through direct protection of neurons. Previous studies have indicated that blocking the EP3 receptor with L798106 rescued the expression of synaptic plasticity-related proteins in an animal model of surgery-induced memory deficits[45].

\section{Conclusions}

In conclusion, our study revealed that the deletion of the EP3 gene can significantly improve the overexpression of ECM in cerebral small arteries of CSVD rats, leading to improved CBF and cognitive function, and the effect may partially through downregulated the TGF- $\beta 1$ /Smad signaling. Thus, blockade of the EP3 receptor may be a promising strategy for the treatment of CSVD. 


\section{Abbreviations}

\begin{tabular}{|ll|}
\hline CSVD & cerebral small vessel disease \\
\hline ECM & extracellular matrix \\
\hline CBF & cerebral blood flow \\
\hline COX-2 & cyclooxygenase 2 \\
\hline PGE 2 & prostaglandins E 2 \\
\hline EP3 & E prostanoid 3 \\
\hline RHRsp & stroke-prone renovascular hypertensive rat \\
\hline aSMA & smooth muscle actin alpha \\
\hline BMVSMCs & brain microvascular smooth muscle cells \\
\hline TGF- $\beta 1$ & transforming growth factor beta1 \\
\hline ANGII & angiotensin II \\
\hline BP & blood pressure \\
\hline
\end{tabular}

\section{Declarations}

\section{Ethics approval and consent to participate}

All animal use procedures were strictly performed in accordance with the Provision and General Recommendation of Chinese Experimental Animals Administration Legislation and were approved by the Fudan University Experimental Animal Science Department Animal Welfare and Ethics Review Board.

\section{Consent for publication}

Not applicable.

\section{Availability of data and materials}

Please contact the author for data requests.

\section{Competing interests}

The authors declare that they have no competing interests.

\section{Funding}

Funding support was provided by the National Natural Science Foundation of China (81671151, Prof Fu), the National Natural Science Foundation of China (81901179, Dr Tang), the National Key Research and 
Development Programs (2016YFC1300600, Profs Fu and Mok); the National Key Research and Development Programs(2016YFC1301603, Prof Fu).

Authors' contributions

FJH, WYL and MV conceived the study and designed experiments. LN and TJ performed the experiments and wrote the manuscript. LN, TJ, XY, ZMY and RX analyzed the results. All authors read and approved the final version of the manuscript.

Acknowledgements

Not applicable.

\section{References}

1. Pantoni L: Cerebral small vessel disease: from pathogenesis and clinical characteristics to therapeutic challenges. Lancet Neurol 2010, 9:689-701.

2. Novak V, Chowdhary A, Farrar B, Nagaraja H, Braun J, Kanard R, Novak P, Slivka A: Altered cerebral vasoregulation in hypertension and stroke.Neurology 2003, 60:1657-1663.

3. De Ciuceis C, Cornali C, Porteri E, Mardighian D, Pinardi C, Fontanella MM, Rodella LF, Rezzani R, Rizzoni D, Boari GE, et al: Cerebral small-resistance artery structure and cerebral blood flow in normotensive subjects and hypertensive patients.Neuroradiology 2014, 56:1103-1111.

4. de la Torre JC: Critically attained threshold of cerebral hypoperfusion: the CATCH hypothesis of Alzheimer's pathogenesis.Neurobiol Aging 2000, 21:331-342.

5. Poels MM, Ikram MA, Vernooij MW, Krestin GP, Hofman A, Niessen WJ, van der Lugt A, Breteler MM: Total cerebral blood flow in relation to cognitive function: the Rotterdam Scan Study.J Cereb Blood Flow Metab 2008, 28:1652-1655.

6. Cannistraro RJ, Badi M, Eidelman BH, Dickson DW, Middlebrooks EH, Meschia JF: CNS small vessel disease: A clinical review.Neurology 2019, 92:1146-1156.

7. Group SPSS, Benavente OR, Coffey CS, Conwit R, Hart RG, McClure LA, Pearce LA, Pergola PE, Szychowski JM: Blood-pressure targets in patients with recent lacunar stroke: the SPS3 randomised trial.Lancet 2013, 382:507-515.

8. Pearce LA, McClure LA, Anderson DC, Jacova C, Sharma M, Hart RG, Benavente OR, Investigators SPS: Effects of long-term blood pressure lowering and dual antiplatelet treatment on cognitive function in patients with recent lacunar stroke: a secondary analysis from the SPS3 randomised trial.Lancet Neurol 2014, 13:1177-1185.

9. Low A, Mak E, Rowe JB, Markus HS, O'Brien JT: Inflammation and cerebral small vessel disease: A systematic review.Ageing Res Rev 2019, 53:100916.

10. Chen GZ, Shan XY, Cheng GP, Tao HM: Cyclooxygenase-2 genetic polymorphism and stroke subtypes in Chinese.J Mol Neurosci 2013, 51:467-473. 
11. Tang J, Xiao W, Li Q, Deng Q, Chu X, Chen Y, Pan D, Fu J: A Cyclooxygenase-2 Inhibitor Reduces Vascular Wall Thickness and Ameliorates Cognitive Impairment in a Cerebral Small Vessel Diseases Rat Model.Curr Alzheimer Res 2015, 12:704-710.

12. Yuhki K, Kojima F, Kashiwagi H, Kawabe J, Fujino T, Narumiya S, Ushikubi F: Roles of prostanoids in the pathogenesis of cardiovascular diseases: Novel insights from knockout mouse studies.Pharmacol Ther 2011, 129:195-205.

13. Yao C, Narumiya S: Prostaglandin-cytokine crosstalk in chronic inflammation.Br J Pharmacol 2019, 176:337-354.

14. Liu B, Zhan M, Zhang Y, Li H, Wu X, Zhuang F, Luo W, Zhou Y: Increased role of E prostanoid receptor3 in prostacyclin-evoked contractile activity of spontaneously hypertensive rat mesenteric resistance arteries.Sci Rep 2017, 7:8927.

15. Avendano MS, Martinez-Revelles S, Aguado A, Simoes MR, Gonzalez-Amor M, Palacios R, GuillemLlobat P, Vassallo DV, Vila L, Garcia-Puig J, et al: Role of COX-2-derived PGE2 on vascular stiffness and function in hypertension. Br J Pharmacol 2016, 173:1541-1555.

16. Lu A, Zuo C, He Y, Chen G, Piao L, Zhang J, Xiao B, Shen Y, Tang J, Kong D, et al: EP3 receptor deficiency attenuates pulmonary hypertension through suppression of Rho/TGF- $\beta 1$ signaling.J Clin Invest 2015, 125:1228-1242.

17. Zeng J, Zhang Y, Mo J, Su Z, Huang R: Two-kidney, two clip renovascular hypertensive rats can be used as stroke-prone rats.Stroke 1998, 29:1708-1713; discussion 1713-1704.

18. Fan Y, Lan L, Zheng L, Ji X, Lin J, Zeng J, Huang R, Sun J: Spontaneous white matter lesion in brain of stroke-prone renovascular hypertensive rats: a study from MRI, pathology and behavior.Metab Brain Dis 2015, 30:1479-1486.

19. Lin J, Lan L, Wang D, Qiu B, Fan Y: Cerebral Venous Collagen Remodeling in a Modified White Matter Lesions Animal Model.Neuroscience 2017, 367:72-84.

20. Bailey EL, Wardlaw JM, Graham D, Dominiczak AF, Sudlow CL, Smith C: Cerebral small vessel endothelial structural changes predate hypertension in stroke-prone spontaneously hypertensive rats: a blinded, controlled immunohistochemical study of 5- to 21-week-old rats.Neuropathol App/ Neurobio/ 2011, 37:711-726.

21. Diglio CA, Grammas P, Giacomelli F, Wiener J: Rat cerebral microvascular smooth muscle cells in culture.J Cell Physio/ 1986, 129:131-141.

22. Gauthier SA, Sahoo S, Jung SS, Levy E: Murine cerebrovascular cells as a cell culture model for cerebral amyloid angiopathy: isolation of smooth muscle and endothelial cells from mouse brain. In Amyloid Proteins. Volume 849. Edited by Sigurdsson E CMGM: Humana Press; 2012: 261-274:

Methods in molecular biology (Clifton, N.J.)].

23. Francois H, Coffman TM: Prostanoids and blood pressure: which way is up? J Clin Invest 2004, 114:757-759.

24. Savoia C, Burger D, Nishigaki N, Montezano A, Touyz RM: Angiotensin II and the vascular phenotype in hypertension. Expert Rev Mol Med 2011, 13:e11. 
25. Liao SJ, Huang RX, Su ZP, Zeng JS, Mo JW, Pei Z, Li L, Fang YN, Hong H, Huang HW: Stroke-prone renovascular hypertensive rat as an animal model for stroke studies: From artery to brain.Journal of the Neurological Sciences 2013, 334:1-5.

26. Ruiz-Ortega M, Rodriguez-Vita J, Sanchez-Lopez E, Carvajal G, Egido J: TGF-beta signaling in vascular fibrosis.Cardiovasc Res 2007, 74:196-206.

27. Sugimoto Y, Narumiya S: Prostaglandin E receptors.J Biol Chem 2007, 282:11613-11617.

28. Muller M, van der Graaf Y, Visseren FL, Mali WP, Geerlings MI, Group SS: Hypertension and longitudinal changes in cerebral blood flow: the SMART-MR study.Ann Neurol 2012, 71:825-833.

29. Saleem S, Kim YT, Maruyama T, Narumiya S, Dore S: Reduced acute brain injury in PGE2 EP3 receptor-deficient mice after cerebral ischemia.J Neuroimmunol 2009, 208:87-93.

30. Ikeda-Matsuo Y, Tanji H, Narumiya S, Sasaki Y: Inhibition of prostaglandin E2 EP3 receptors improves stroke injury via anti-inflammatory and anti-apoptotic mechanisms.J Neuroimmunol 2011, 238:34-43.

31. Wong SM, Jansen JFA, Zhang CE, Hoff El, Staals J, van Oostenbrugge RJ, Backes WH: Blood-brain barrier impairment and hypoperfusion are linked in cerebral small vessel disease.Neurology 2019, 92:e1669-e1677.

32. Facemire CS, Griffiths R, Audoly LP, Koller BH, Coffman TM: The impact of microsomal prostaglandin e synthase 1 on blood pressure is determined by genetic background. Hypertension 2010, 55:531-538.

33. Xiao L, Itani HA, do Carmo LS, Carver LS, Breyer RM, Harrison DG: Central EP3 (E Prostanoid 3) Receptors Mediate Salt-Sensitive Hypertension and Immune Activation.Hypertension 2019, 74:15071515.

34. Chen L, Miao Y, Zhang Y, Dou D, Liu L, Tian X, Yang G, Pu D, Zhang X, Kang J, et al: Inactivation of the E-prostanoid 3 receptor attenuates the angiotensin II pressor response via decreasing arterial contractility.Arterioscler Thromb Vasc Bio/ 2012, 32:3024-3032.

35. Zhang J, Zou F, Tang J, Zhang Q, Gong Y, Wang Q, Shen Y, Xiong L, Breyer RM, Lazarus M, et al: Cyclooxygenase-2-derived prostaglandin EX promotes injury-induced vascular neointimal hyperplasia through the E-prostanoid 3 receptor. Circ Res 2013, 113:104-114.

36. Torun D, Ozelsancak R, Turan I, Micozkadioglu H, Sezer S, Ozdemir FN: The relationship between obesity and transforming growth factor beta on renal damage in essential hypertension.International Heart Journal 2007, 48:733-741.

37. Jing L, Zhang JZ, Zhao L, Wang YL, Guo FY: High-expression of transforming growth factor beta1 and phosphorylation of extracellular signal-regulated protein kinase in vascular smooth muscle cells from aorta and renal arterioles of spontaneous hypertension rats. Clin Exp Hypertens 2007, 29:107117.

38. Thibodeau JF, Nasrallah R, Carter A, He Y, Touyz R, Hebert RL, Kennedy CRJ: PTGER1 deletion attenuates renal injury in diabetic mouse models. Am J Pathol 2013, 183:1789-1802.

39. Jensen MS, Mutsaers HAM, Tingskov SJ, Christensen M, Madsen MG, Olinga P, Kwon TH, Norregaard R: Activation of the prostaglandin E2 EP2 receptor attenuates renal fibrosis in unilateral ureteral 
obstructed mice and human kidney slices.Acta Physiol (Oxf) 2019, 227:e13291.

40. Thibodeau JF, Holterman CE, He Y, Carter A, Cron GO, Boisvert NC, Abd-Elrahman KS, Hsu KJ, Ferguson SS, Kennedy CR: Vascular Smooth Muscle-Specific EP4 Receptor Deletion in Mice Exacerbates Angiotensin II-Induced Renal Injury.Antioxid Redox Signal 2016, 25:642-656.

41. Zhao J, Shu B, Chen L, Tang J, Zhang L, Xie J, Liu X, Xu Y, Qi S: Prostaglandin E2 inhibits collagen synthesis in dermal fibroblasts and prevents hypertrophic scar formation in vivo.Exp Dermato/ 2016, 25:604-610.

42. van Rodijnen WF, Korstjens IJ, Legerstee N, Ter Wee PM, Tangelder GJ: Direct vasoconstrictor effect of prostaglandin E2 on renal interlobular arteries: role of the EP3 receptor.Am J Physiol Renal Physiol 2007, 292:F1094-1101.

43. Jadhav V, Jabre A, Lin SZ, Lee TJ: EP1- and EP3-receptors mediate prostaglandin E2-induced constriction of porcine large cerebral arteries.J Cereb Blood Flow Metab 2004, 24:1305-1316.

44. Shum WW, Le GY, Jones RL, Gurney AM, Sasaki Y: Involvement of Rho-kinase in contraction of guinea-pig aorta induced by prostanoid EP3 receptor agonists. Br J Pharmacol 2003, 139:1449-1461.

45. Xiao JY, Xiong BR, Zhang W, Zhou WC, Yang H, Gao F, Xiang HB, Manyande A, Tian XB, Tian YK: PGE2-EP3 signaling exacerbates hippocampus-dependent cognitive impairment after laparotomy by reducing expression levels of hippocampal synaptic plasticity-related proteins in aged mice.CNS Neurosci Ther 2018, 24:917-929.

\section{Figures}


A

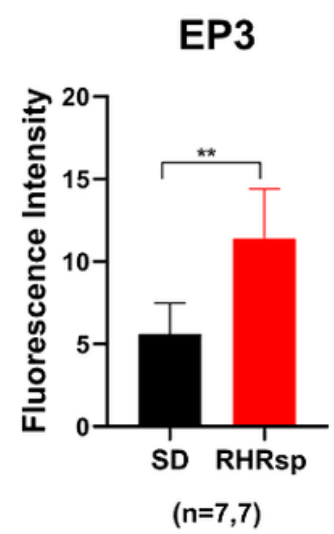

EP3
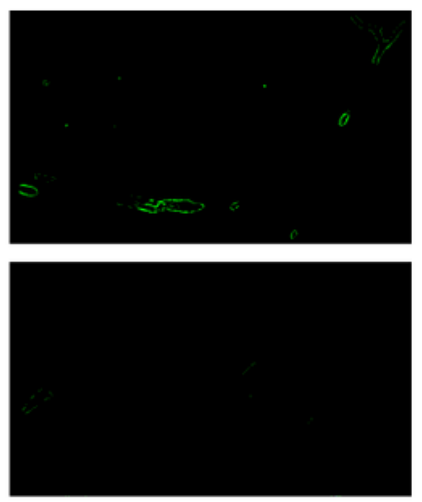

aSMA
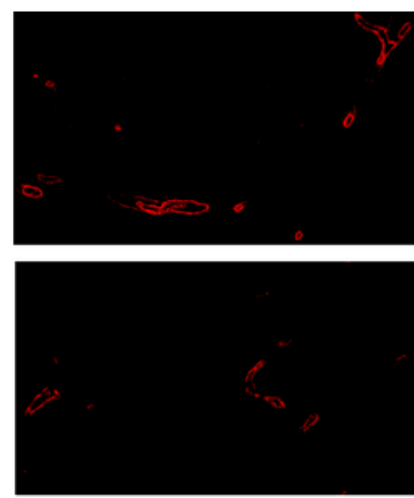

Merge
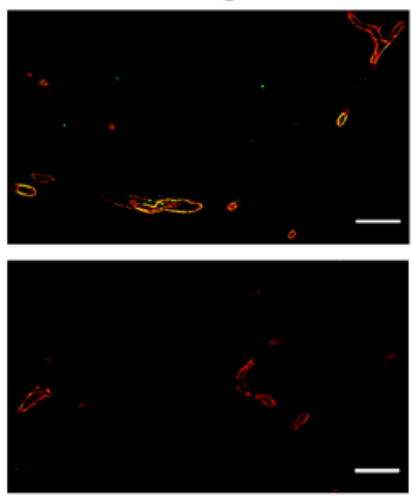

B BP (Non-Invasive)

Invasive BP

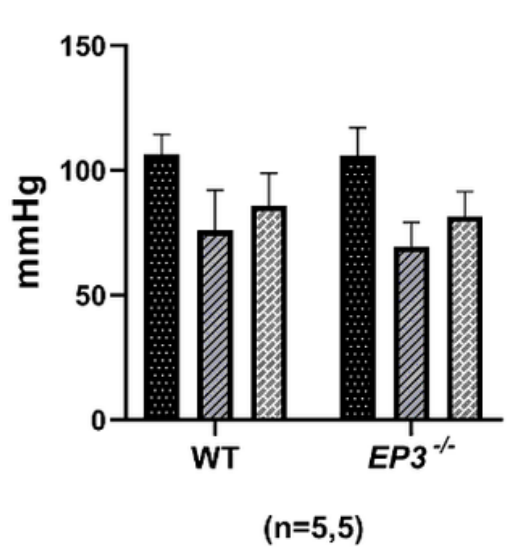

BP (Non-Invasive)

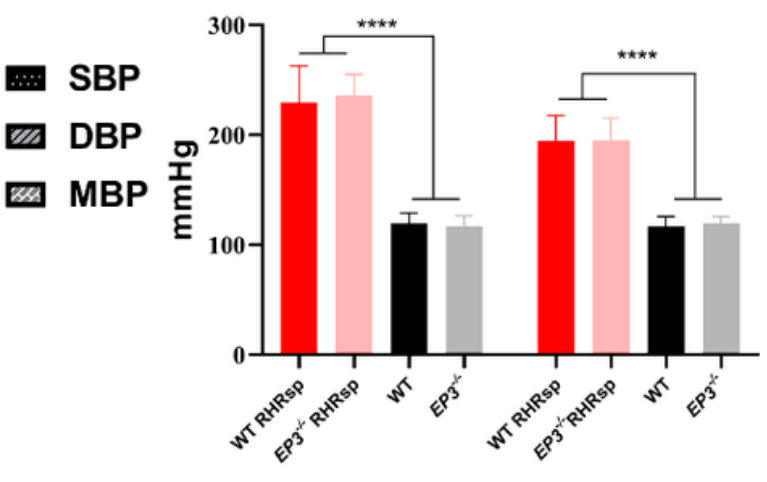

$2 \mathrm{~m}$ post-operation $4 \overline{\mathrm{m} \text { post-operation }}$ $(n=9,9,6,6 ; n=8,8,6,6)$

Figure 1

(A) Immunofluorescence for EP3 (Green) and aSMA (Red) of SD and RHRsp. The cerebral small arteries $(10-65 \mu \mathrm{m})$ are indicated by the immunostaining of aSMA. Bar, 100um. (B)Blood pressure in SD and RHRsp. SD, Sprague Dawley rat; RHRsp, Stroke-prone renovascular hypertensive rat; aSMA, smooth muscle actin alpha; BP, blood pressure; SBP, systolic blood pressure; DBP, diastolic blood pressure; MBP,

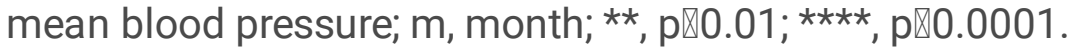


A

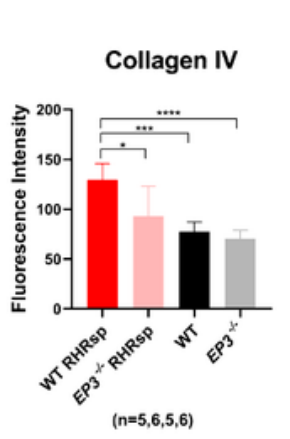

B

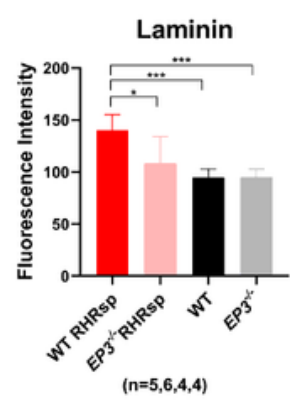

C

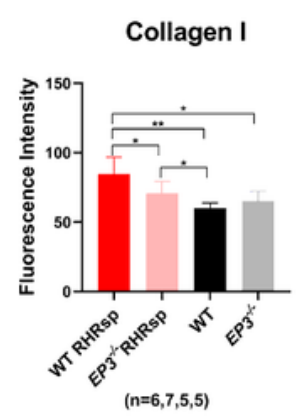

D

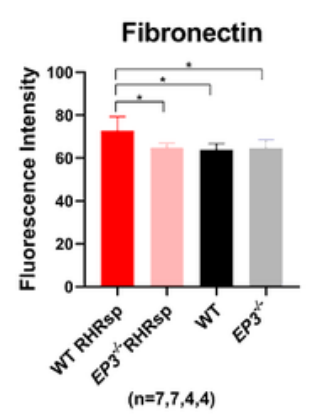

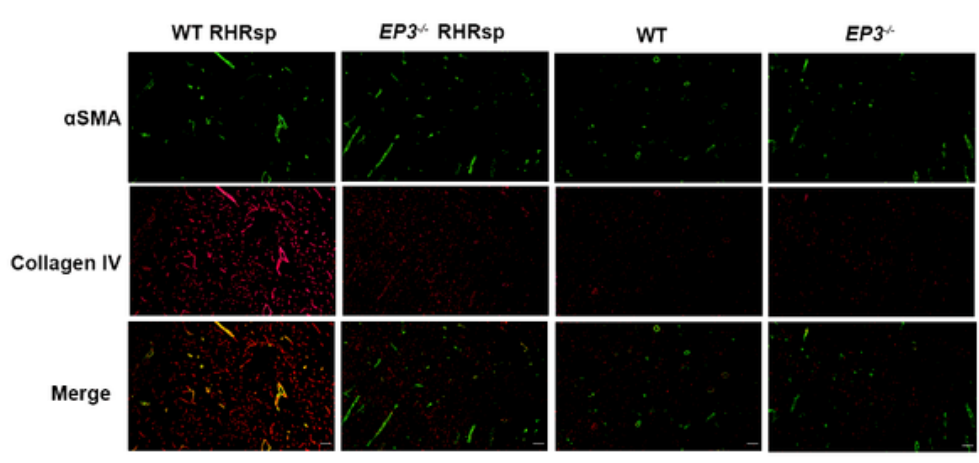
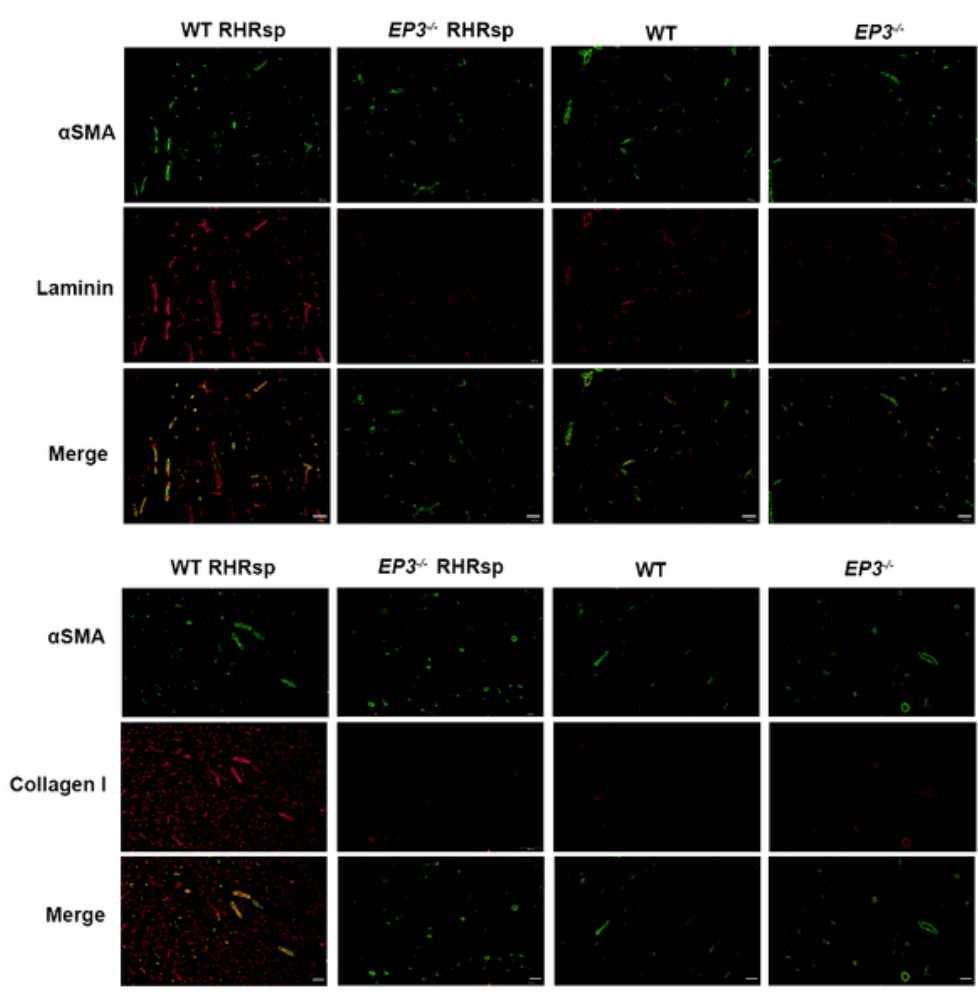

EP3 RHRsp
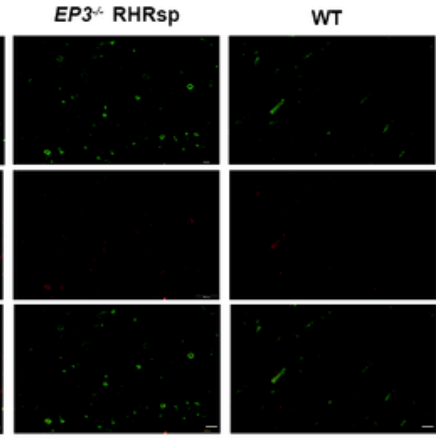

EP3

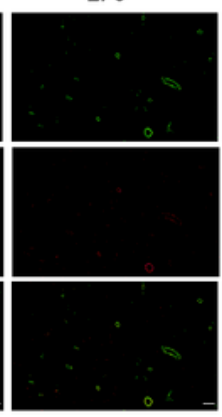

EP3 RHRsp
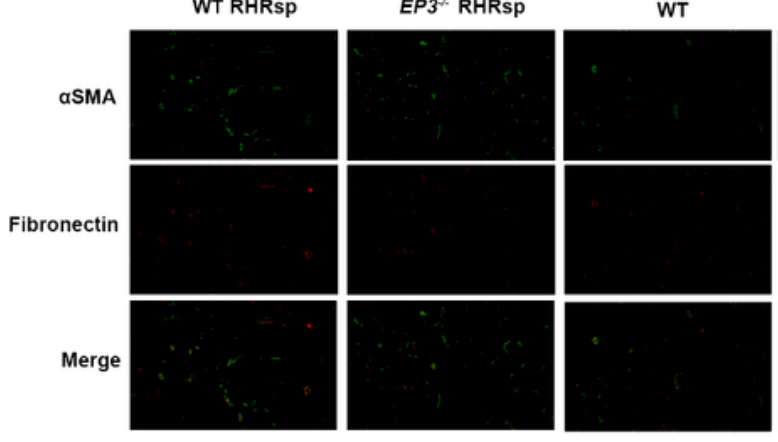

$E P 3^{-}$

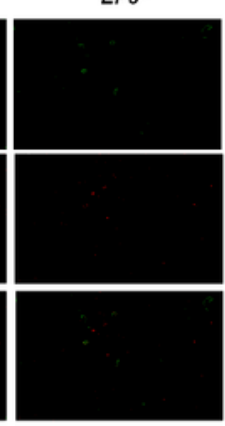

\section{Figure 2}

The deposition of extracellular matrix in the vessel wall of cerebral small arteries. (A)Immunofluorescence for aSMA (Green) and collagen IV (Red) of the animals. (B) Immunofluorescence for aSMA (Green) and laminin (Red) of the animals; (C) Immunofluorescence for aSMA (Green) and collagen I (Red) of the animals ; (D) Immunofluorescence for aSMA (Green) and fibronectin (Red) of the animals. The cerebral small arteries (10-65 $\mu \mathrm{m})$ are indicated by the immunostaining of aSMA. Bar, 100um. RHRsp, Stroke-prone 


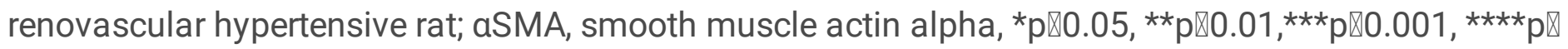
0.001 .
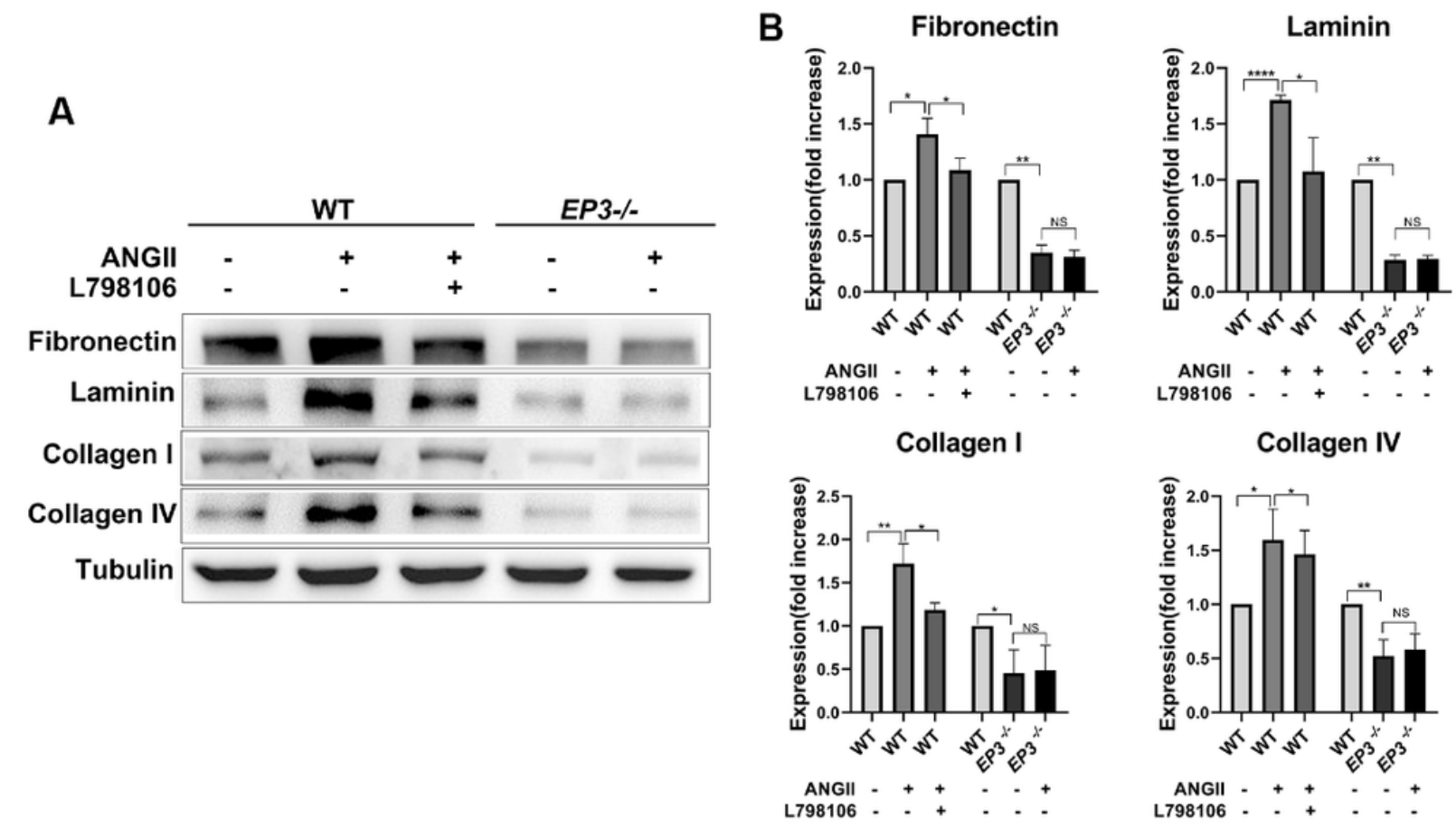

Figure 3

Change of extracellular matrix expression in rat BMVSMCs under different stimulation. (A)Representative western blots and quantification of the expression of fibronectin, laminin, collagen I and collagen IV in rat BMVSMCs under different stimulation. (B) Quantification of western blots of fibronectin, laminin, collagen I and collagen IV. BMVSMCs, brain microvascular smooth muscle cells; ANGII, Angiotensin II. *, $p$ $\varangle 0.05 ; * \star, p \otimes 0.01 ; * \star \star \star, ~ p \otimes 0.0001$. 
A
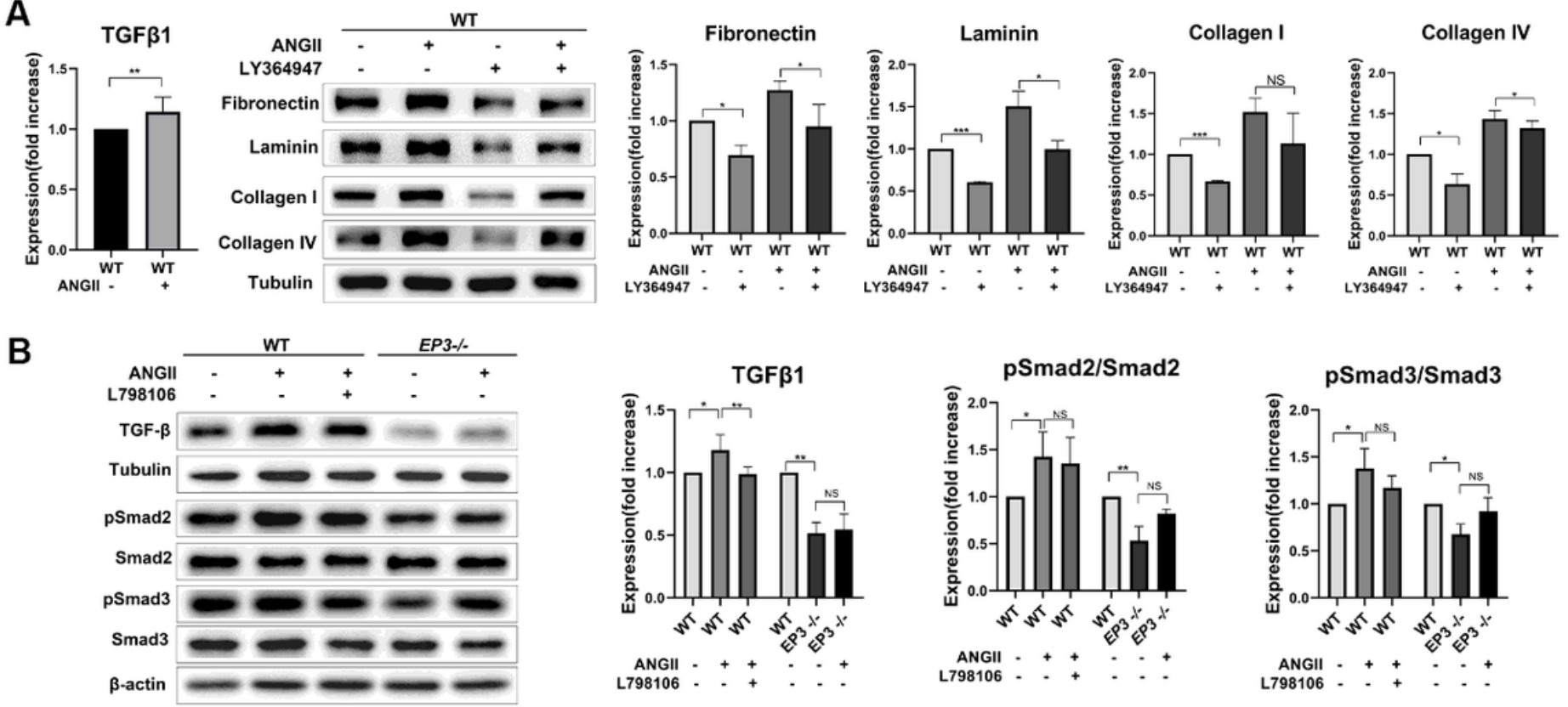

C
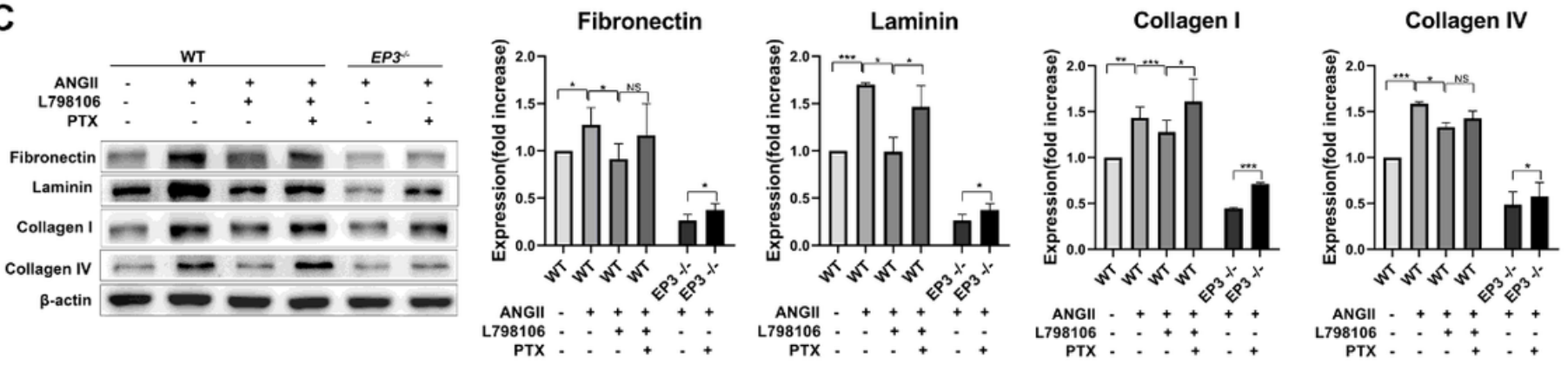

\section{Figure 4}

Quantification of western analysis. (A) TGF- $\beta 1$ were elevated in rat BMVSMCs under ANGII stimulation. Suppressing TGF- $\beta 1$ by LY364947 reversed the high expression of ECM in rat BMVSMCs under ANGII stimulation. (B) Deletion of EP3 rescued ANGIl-induced activation of TGF- $\beta 1 /$ SMAD2/signaling. (C) The downregulation of ECM by the inhabitation of EP3 was abolished by pretreatment with PTX in BMVSMCs. BMVSMCs, brain microvascular smooth muscle cells; ECM, extracellular matrix; TGF- $\beta 1$,

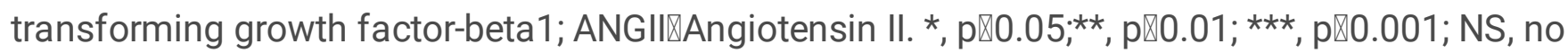
significant statistical difference. 
A

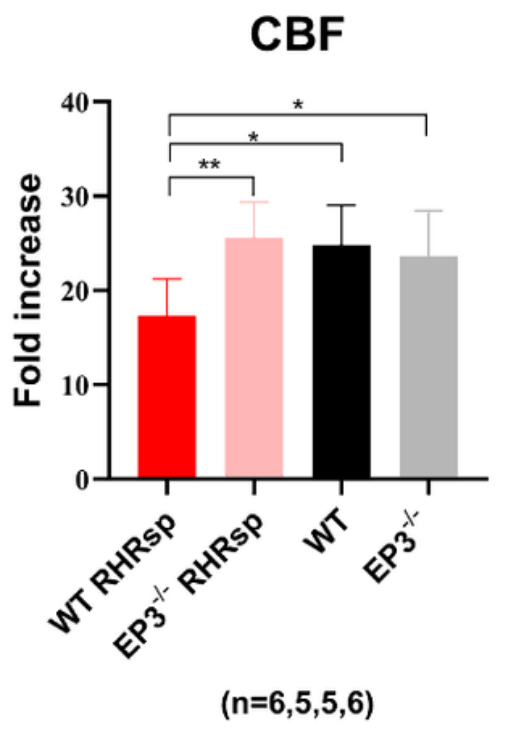

B

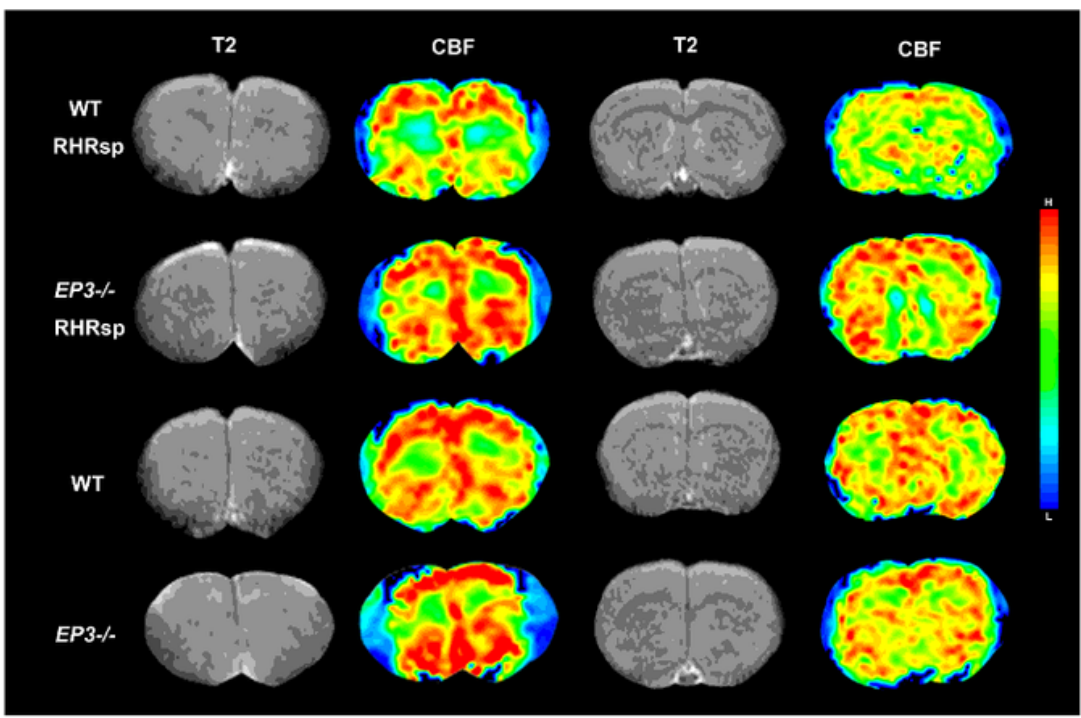

Figure 5

(A) Quantitative results of CBF. (B) Representative picture of CBF. CBF, cerebral blood flow; RHRsp, Stroke-

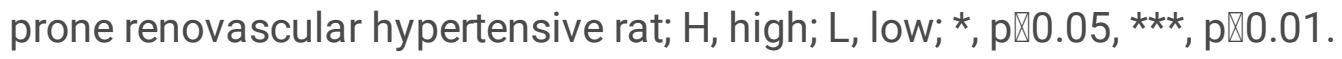


A

Escape Latency

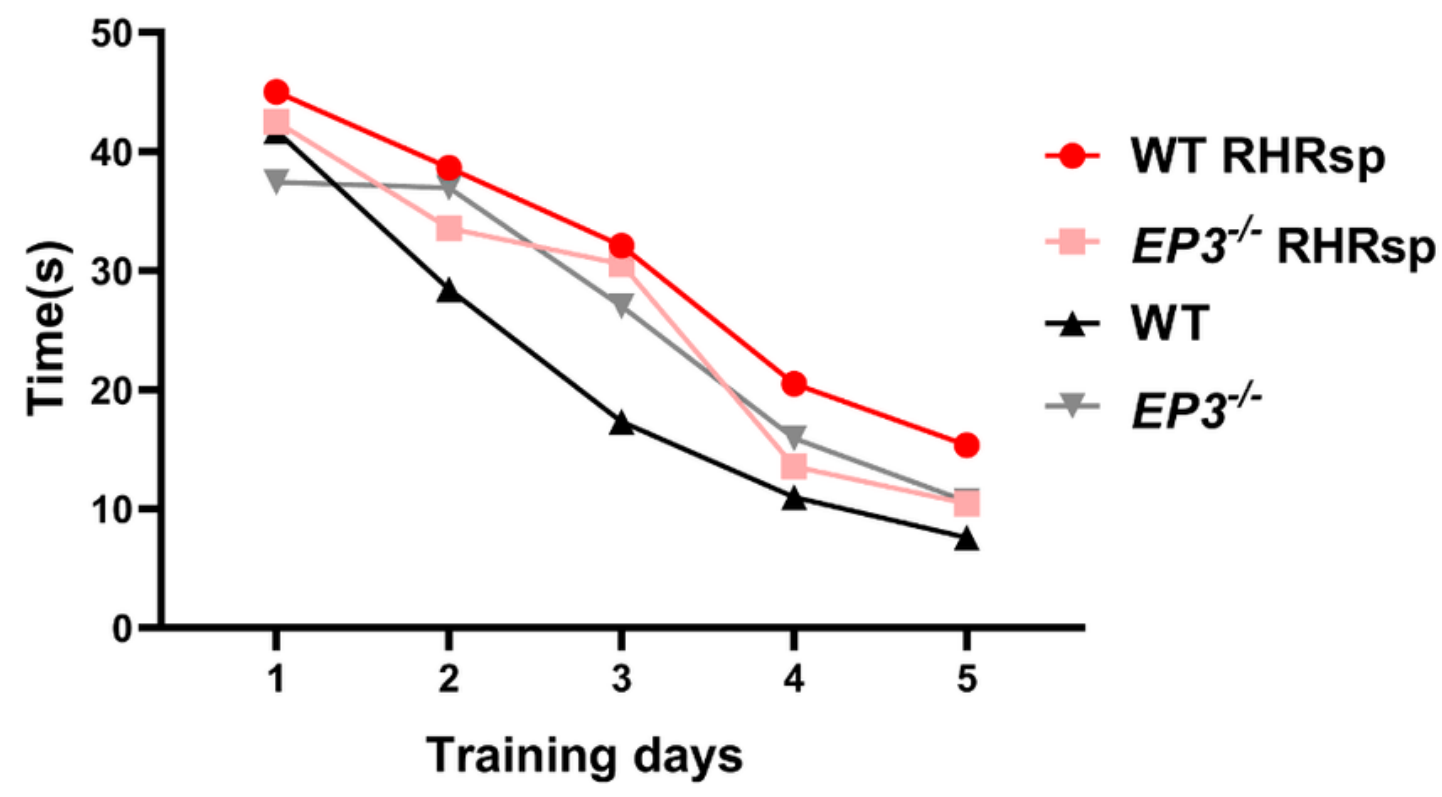

B

\section{Time Spent in the Target Quadrant}

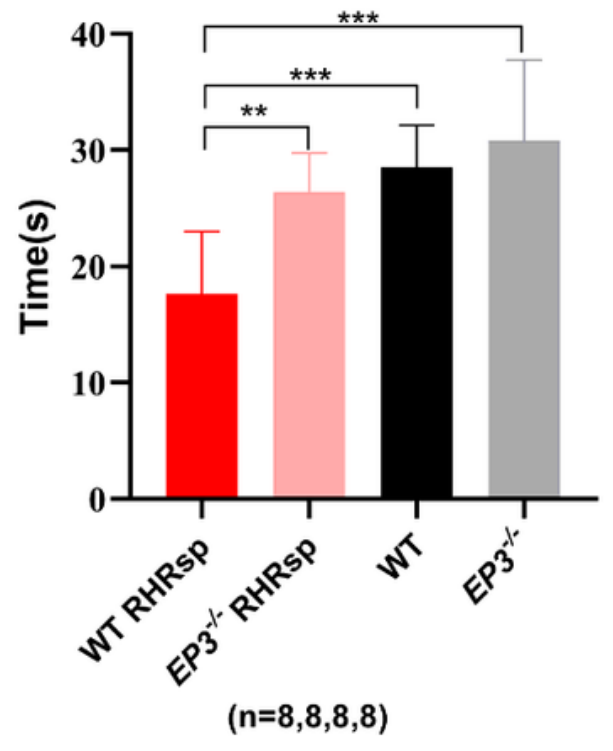

Number of Times Crossing the Platform

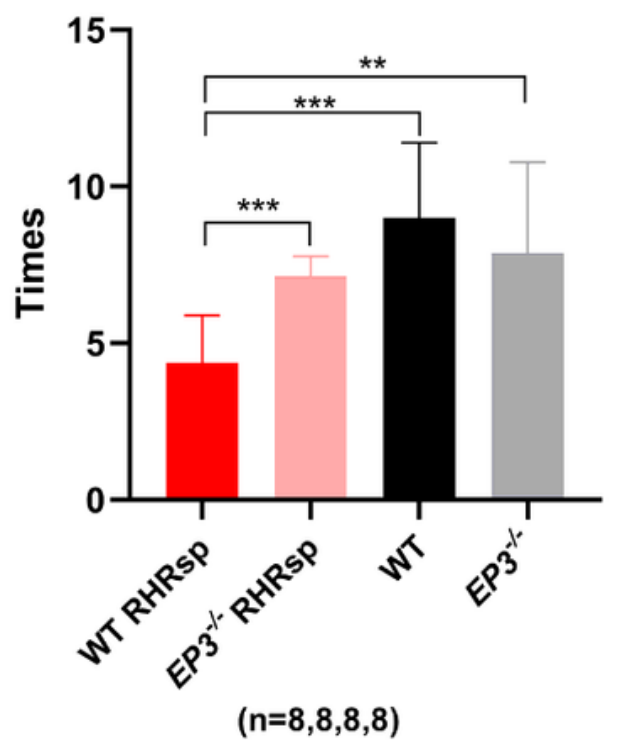

Figure 6

(A) Morris water maze test: escape latency in place navigation test. (B) Morris water maze test: time spent in the target quadrant in the spatial probe test. (C) Morris water maze test: the number of times

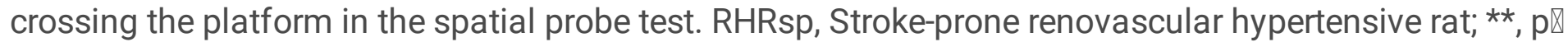
$0.01 ; * \star \star, p \llbracket 0.001$. 


\section{Supplementary Files}

This is a list of supplementary files associated with this preprint. Click to download.

- SupplementalMaterial.docx 